\title{
DETERMINACIÓN DE FUNCIONES DE VOLUMEN PARA EUCALIPTO
}

\author{
JUAN CARLOS PINILLA SUÁREZ y CARLOS KAHLER GONZÁLEZ \\ Ingenieros Forestales, División Ordenación Forestal e Inventarios. Instituto Forestal
}

\section{RESUMEN}

Dentro de las actividades del Proyecto "Manejo Silvicola de Especies del Género Eucalyptus" se desarnollaron funciones de volumen para eucalipto. Los datos para su construcción provinieron de rodales adultos de Eucalyptus globulus ubicados en la $V$, Vl y $\mathrm{X}$ Región de Chile, obteniendo la información luego de derribar y medir los árboles. Se utilizó el ajuste de un modelo matemático según área geográfica $y$ un modelo a nivel general. Al analizar los resultados se concluve que el modelo de Spurr desarrollado para cada localidad seria recomendable para ser empleado. Los modelos obtenidos son confiables en sus estimaciones. pero se debe considerar que su construcción se basa en información proveniente de rodales adultos de eucalipto. Para desarrollar modelos más eficientes se necesita aumentar la base de datos empleada en su construcción atendiendo las necesidades de manejo para eucalipto.

Palabras Clave: Eucalyptus. Volumen.

\section{ABSTRACT}

One of the activities in "Forestry Management of the Eucaliptus Species Project" was to develop volume functions. The data was taken from adults stands of Eucaliptus globulus located in the IV VI and X Regions of Chile. Trees were harvested in order to measure them. It was used a mathematical model according to the geographical area and a general one. After analyzing the results we concluded that the Spurr mathematical model developed for every zone is the most appropriate to evaluate the tree volumes. The models presents reliable estimate but it should he taken into account that the data comes from adult Eucalyptus globulus stands. The development of more efficient models will depend on a larger data set and the Eucalyptus management requirements.

Key words: Eucalyptus. Volume. 


\section{INTRODUCCIÓN}

Para la cuantificación de los productos forestales que el bosque puede proporcionar se recurre, principalmente, a la determinación del volumen de los árboles o parte de él. siendo este parámetro una de las informaciones importantes en la descripción de las masas forestales. De igual modo, una de las formas más utilizadas para la evaluación de la productividad de un sitio es la deterininación del volumen, ya que este valor permite apreciar la ganancia producida al combinar los efectos del diámetro y la altura.

Existen numerosas formas para la determinación del volumen de un árbol, siendo las más utilizadas aquellas que relacionan variables dasométricas con los distintos tipos de volumen presentes en un árbol. En la búsqueda de estas relaciones se han desarrollado modelos matemáticos que utilizan como variable dependiente el volumen. y como independientes. variables de estado del árbol fáciles de medir. como son el diámetro a la altura del pecho (DAP) y la altura total y/o alguna otra sencilla de cuantificar, asi como también transformaciones de ellas.

En este sentido. un buen modelo o función de volumen debería cumplir con ser suficientemente flexible. compatible con funciones de ahusamiento o funciones de volumen de trozas. buen estimador de dicha variable, simple en su expresión analítica. de fácil aplicación y/o costo y ser lógico en su formulación. La literatura señala. además. que los modelos simples en empleo y construcción evidencian buenas propiedades estimadoras.

Dada la importancia de estas relaciones como herramienta del manejo forestal de las especies de eucalipto de interés para nuestro país, es que el proyecto Manejo Silvícola de Especies del Género Eucalyptus de la División Ordenación Forestal e Inventarios de INFOR. tiene entre sus objetivos el investigar y obtener antecedentes sobre el crecimiento y rendimiento de los rodales de eucalipto. desarrollando los modelos matemáticos más apropiados para ello. Dada la superficie actualmente cubierta con Eucalyptus globulus ssp globulus. es que esta investigación esta orientada principalmente a esta especie.

Un aspecto relevante en este sentido es la obtención de la información desde los rodales de interés. su análisis y utilización para obtener las herramientas que apoyen las decisiones de manejo. optimizando las medidas silviculturales que permitan alcanzar los objetivos de producción.

Como antecedente se puede mencionar que en el pais las empresas forestales que cuentan con eucalipto en su patrimonio han desarrollado algunos modelos de volumen. Sin embargo, la información sobre la utilización de estas funciones en especies del género Eucalyptus no está muỵ difundida o es muy limitada. 
Debido a la falta de antecedentes, el proyecto ha reunido una base de datos que permite determinar funciones de volumen, utilizando la información disponible y métodos y técnicas estadísticas.

El presente trabajo describe lo realizado por el proyecto en el desarrollo de funciones de volumen para eucalipto en edades adultas, presentando un modelo basado en la información actualmente disponible.

\section{DATOS}

La información se obtuvo de la medición de árboles de tres rodales adultos de eucalipto generados a partir de monte alto, ubicados en la V. VI y X Región. pertenecientes a empresas o particulares.

Una característica especial de estos rodales es su edad adulta. Aunque no fue posible obtener la edad precisa de cada uno. como minimo tenian 25 años al momento de su explotación. La ubicación y descripción de los rodales se presentan en el Cuadro $\mathrm{N}^{\mathrm{o}} 1$.

En cada una de las áreas de estudio se utilizó la misma metodología para obtener la información. Esta consistió, en obtener la distribución diamétrica del rodal. Posteriormente se eligió un número de árboles representativos de toda la distribución diamétrica del rodal, valor variable en razón del número de árboles presentes en el rodal. Los árboles seleccionados fueron derribados y medidos en secciones

\section{Cuadro $\mathrm{N}^{\circ} 1$}

DESCRIPCIÓN ÁREAS DE ESTUDIO

\begin{tabular}{|l|c|c|c|}
\hline \multicolumn{1}{|c|}{ VARIABLES } & \multicolumn{3}{c|}{ AREAS DE ESTUDIO } \\
\hline Región & Los Riscos & Tanumé & Peñuelas \\
\hline Sector & $\mathrm{I}$ & $\mathrm{VI}$ & $\mathrm{V}$ \\
\hline Propietario & Puerto Varas & Pichilemu & Lago Peñuelas \\
\hline $\mathbf{N}^{\bullet}$ de árboles & Forestal Los Lagos & CONAF & CONAF \\
\hline $\mathbf{N}^{\bullet}$ de observaciones & 49 & 68 & 70 \\
\hline $\mathbf{N}^{\bullet}$ observaciones/árbol & 1166 & 2208 & 2453 \\
\hline
\end{tabular}

En cada árbol derribado sc midió y registró una serie de variables. las que se detallan a continuación y' en la Figura $\mathrm{N}^{\circ} 1$ :
a) Región.
b) Empresa.
c) Nombre del predio. 
d) Número del árbol.

e) Diámetro a la altura del pecho $(\mathrm{cm})$ con corteza.

f) Diámetros cada $1 \mathrm{~m}$, hasta los $5 \mathrm{~cm}$ de diámetro con corteza.

g) Largos de las secciones medidas (generalmente 1 metro).

h) Altura total (m).

i) Altura de copa (m).

j) Altura de tocón $(\mathrm{cm})$.

k) Diámetro comienzo de copa $(\mathrm{cm})$.

1) Diámetro de tocón $(\mathrm{cm})$.

m) Doble espesor de corteza ( $\mathrm{mm}$ ) en cada sección.

Posteriormente. toda la información fue traspasada a una base de datos para su utilización y manejo en este estudio. La información recolectada fue sometida a un proceso de depuración gráfica y analitica. eliminándose los datos ilógicos o con errores.

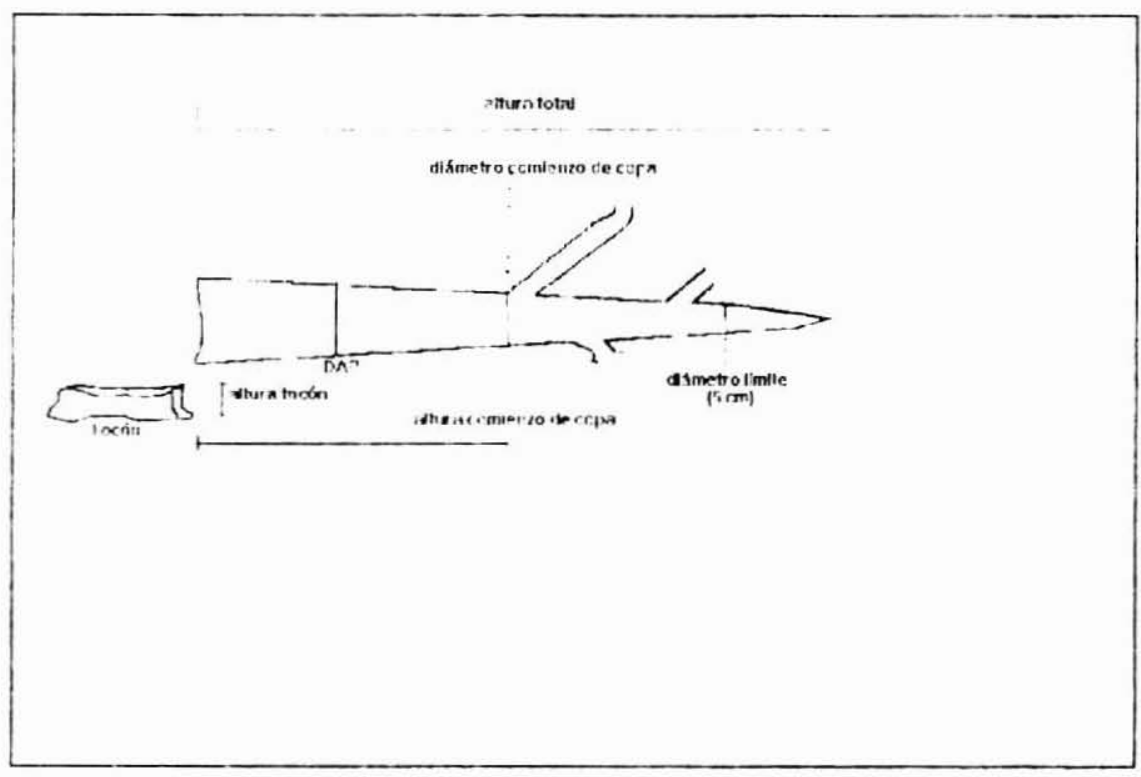

Figura $N^{\circ} 1$. MEDICIONES REALIZADAS EN LOS ÁRBOLES DERRIBADOS

Una descripción de las frecuencias y márgenes de altura por clase diamétrica de los árboles de la muestra. se presenta en la Cuadro $\mathrm{N}^{\circ} 2$. En esta tabla se indican los diámetros superiores de la muestra. y también las alturas de los árboles. 


\section{Cuadro $\mathrm{N}^{2} 2$.}

\section{DISTRIBUCIÓN DE LA MUESTRA SEGÚN CLASE DIAMÉTRICA Y RANGOS DE ALTURA.}

\begin{tabular}{|c|c|c|c|c|c|}
\hline $\begin{array}{c}\text { Clase de } \\
\text { DAP } \\
(\mathrm{cm})\end{array}$ & $\begin{array}{c}\text { Número } \\
\text { de árboles }\end{array}$ & $\begin{array}{c}\text { DAP } \\
\text { minimo } \\
(\mathrm{cm})\end{array}$ & $\begin{array}{c}\text { DAP } \\
\text { máximo } \\
(\mathrm{cm})\end{array}$ & $\begin{array}{c}\text { Altura } \\
\text { minima } \\
(\mathrm{m})\end{array}$ & $\begin{array}{c}\text { Altura } \\
\text { máxima } \\
(\mathbf{m})\end{array}$ \\
\hline$<10$ & 11 & 6,20 & 9,97 & 10,3 & 13,7 \\
\hline $10-20$ & 23 & 11.4 & 19,9 & 13,0 & 25,3 \\
\hline $20-30$ & 27 & 20,0 & 29,4 & 15,0 & 37,0 \\
\hline $30-40$ & 26 & 30,0 & 39,9 & 22,4 & 42,7 \\
\hline $40-50$ & 25 & 40,2 & 49,8 & 29,8 & 48,3 \\
\hline $50-60$ & 23 & 50,0 & 59,0 & 33,4 & 49,0 \\
\hline $60-70$ & 22 & 60,2 & 69,5 & 29,9 & 50,5 \\
\hline $70-80$ & 15 & 70,0 & 79,0 & 30,2 & 51,7 \\
\hline $80-90$ & 10 & 80,0 & 89,4 & 34,0 & 46,6 \\
\hline Total & 182 & & & & \\
\hline
\end{tabular}

\section{MÉTODOS}

Con la información depurada se procedió a realizar una serie de análisis para la estimación de las funciones de volumen. utilizando los datos en forma agrupada o desagregada según región o rodal.

\section{Ajuste de una Función a Nivel de Rodal}

En una primera etapa se ajustaron funciones de volumen a los datos obtenidos. separando la información según el rodal de origen. El ajuste se realizó utilizando el método de los minimos cuadrados.

De acuerdo con la distribución que presentó el volumen en cada una de las áreas de estudio, y agrupados los datos en forma general, y dado los resuitados que se han obtenido con su aplicación. se decidió ajustar el modelo desarrollado por Spurr (1952). cuya fórmula es:

$$
V=a+b^{*} D A P^{2} * H
$$

donde:

$\mathrm{V}=$ Volumen en $\mathrm{m}^{3} \mathrm{ssc}$. desde la altura de tocón hasta la altura de un diámetro límite de $5 \mathrm{~cm}$ con corteza. 
$\mathrm{DAP}=\mathrm{DAP}$ con corteza, en centímetros.

$\mathrm{H}=$ Altura total, en metros.

La aplicación de esta función, permite evaluar las propiedades del modelo al comparar los volúmenes reales medidos con los correspondientes estimados.

\section{Evaluación de la Función}

Como indicadores de la bondad de ajuste de la función de volumen se utilizaron: el error cuadrático medio porcentual $(\operatorname{ECM}(\%))$. como indicador de la exactitud de la función: sesgo probable en porcentaje (DIFA(\%)). como indicador de la magnitud de los sesgos: el estimador de Durbin-Watson (correlación serial de los residuos del modelo). el coeficiente de correlación y el error estándar.

El Error Cuadrático Medio Porcentual (ECM\%) y la Diferencia Agregada Porcentual (DIFA\%) se definen matemáticamente por las siguientes expresiones:

$$
\begin{aligned}
& \operatorname{ECM}(\%)=\left(\sqrt{\frac{\left(\sum(V o-V e)^{2}\right)}{n}}\right) * \frac{100}{Y} \\
& D I F A(\%)=\left(\frac{\sum V O-\sum V e}{\sum V o}\right) * 100
\end{aligned}
$$

donde:

$V_{0}=$ Volumen observado .

$\mathrm{Ve}=$ Volumen estimado

$\mathrm{Y} \quad=$ Volumen observado promedio

$\mathrm{N}=$ Número de árboles

La cubicación de las trozas para la obtención del volumen observado se realizó mediante la fórmula de Smalian (Husch. 1982). 


\section{Ajuste de una Función General de Volumen}

Un segundo tipo de estudio se realizó ajustando un modelo de volumen al total de la información experimental.

El ajuste se realizó utilizando el método de los mínimos cuadrados y por la combinación de variables empleadas en los modelos considerados, usando la técnica de regresión paso a paso

En la evaluación de los modelos de volumen se utilizaron también como indicadores de la bondad de ajuste. el error cuadrático medio porcentual (ECM(\%)), como indicador de la exactitud de la función: sesgo probable en porcentaje (DIFA(\%)), como indicador de la magnitud de los sesgos; el estimador de Durbin-Watson (correlación serial de los residuos del modelo). el coeficiente de correlación y el error estándar.

\section{RESULTADOS}

Utilizando técnicas estadísticas y observando como se dispersan gráficamente las estimaciones entregadas por las funciones de volumen ajustadas, en relación a los datos originales. fue posible establecer las tendencias de las funciones por utilizar y realizar las estimaciones de los distintos indicadores de la bondad de los ajustes.

Se uso en este proceso la información básica entregada por el muestreo. luego de su depuración. utilizando las variables originales y sus combinaciones.

\section{Ajuste de la Función de Volumen a Nivel Local}

La elección del modelo a ajustar tuvo por objetivo compatibilizar la necesidad de obtener un modelo lo más semejante a la situación real (los modelos que representan sistemas biológicos generalmente son complejos). $\mathrm{y}$ la simplicidad en las mediciones de las variables requeridas $y$ los costos involucrados. va que a mayor número de parámetros a estimar en terreno. mayor costo de la información base $y$ su procesamiento

La distribución del volumen observado en el área de Los Riscos. Tanumé y Peñuelas se presenta en las figuras $\mathrm{N}^{\circ}$ 2. 3. y 4. respectivamente. Al analizar gráficamente la distribución de estos volúmenes en relación al DAP de los árboles. fue posible apreciar que éstos se agrupaban de acuerdo con una tendencia común.

Una vez realizados los ajustes según área de estudio. se obtuvieron los respectivos coeficientes de las funciones y sus indicadores de la bondad de ajuste. los cuales son presentados en el Cuadro $\mathrm{N}^{\circ} 3$. 


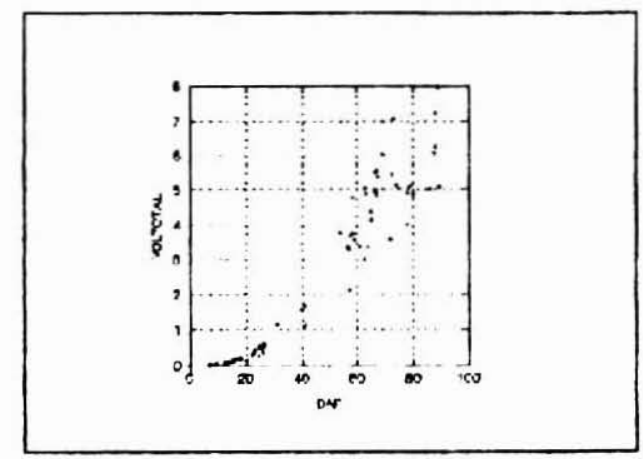

Figura $N^{\circ}$ 2. RELACIÓN DAP/NOLUMEN AREA LOS RISCOS

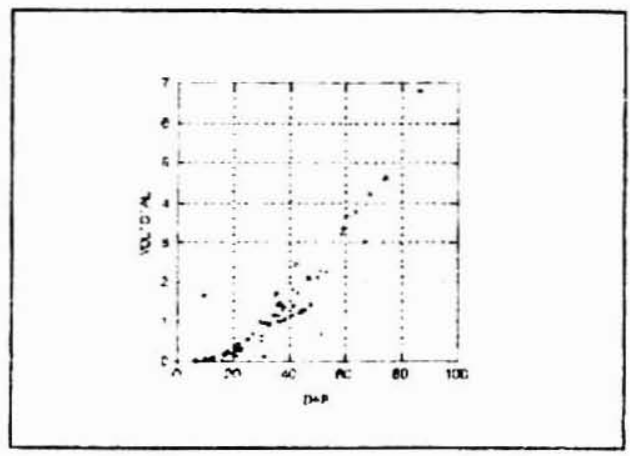

Figura $N^{\circ}$ 3. RELACIÓN DAPNOLUMEN ÁREA TANUMÉ

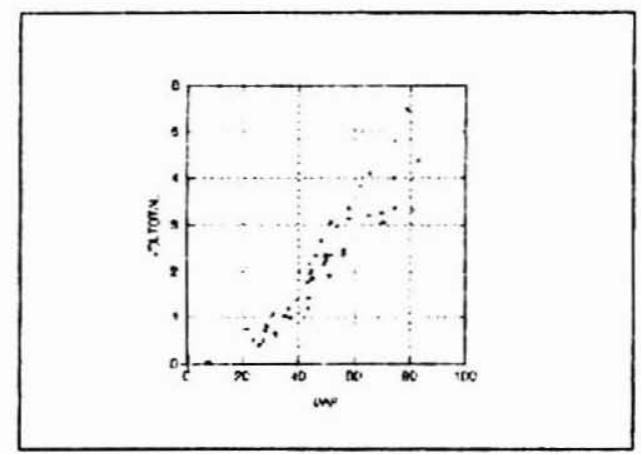

Figura No 4. RELACIÓN DAPNOLUMEN ÁREA PENUULLAS

Los valores obtenidos en los ajustes (Cuadro $\mathrm{N}^{\circ} 3$ ), permiten concluir que la ecuación de Spurr parece representar razonablemente bien el comportamiento 


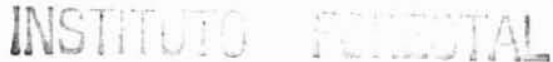

volumétrico en las tres áreas en estudio. En efecto, el valor de los coeficientes de correlación sefialan un alto ajuste entre los puntos de las tres muestras y los modelos obtenidos. Junto con ello el error estándar, el cual indica la dispersión de los puntos con respecto de los modelos obtenidos, no fue superior a 0,528 (área Tanumé). llegando a 0,048 en el área de Los Riscos. Por otra parte, los valores del estimador de DurbinWatson, referidos a la correlación serial de los residuos del modelo, están en el margen que indica que no existe una autocorrelación, presentando el área de Tanumé el menor valor $(1,5)$.

\section{Cuadro $\mathrm{N}^{\circ} 3$.}

COEFICIENTES DE FUNCIONES E INDICADORES DEL A.JUSTE

\begin{tabular}{|l|c|c|c|}
\hline \multirow{2}{*}{ COEFICIENTES } & \multicolumn{3}{|c|}{ AREAS DE ESTUDIO } \\
\cline { 2 - 4 } & Tanume & Peñuelas & Los Riscos \\
\hline $\mathrm{a}$ & 0,1976789 & 0,2490222 & 0,0195213 \\
\hline $\mathrm{b}$ & 0.0000212 & 0,0000194 & 0.0000282 \\
\hline $\mathrm{r}$ & 0,98 & 0,98 & 0,99 \\
\hline $\mathrm{E} . \mathrm{S}$ & 0,528 & 0,266 & 0,048 \\
\hline $\mathrm{DW}$ & 1.5 & 1,9 & 2,2 \\
\hline $\mathrm{ECM}\left(\mathrm{m}^{3}\right)$ & 0,5198 & 0,2622 & 0,0463 \\
\hline $\mathrm{ECM}(\%)$ & 17,33 & 12,47 & 8,14 \\
\hline $\mathrm{DIFA}(\%)$ & 0.2027 & $-0,0034$ & $-0,0768$ \\
\hline $\mathrm{N}^{\circ}$ de arboles & 65 & 65 & 42 \\
\hline $\mathrm{F}_{\text {cal }}$ & $1.326 .82^{\circ}$ & $1.481,84^{\circ}$ & $5.426 .82^{\circ}$ \\
\hline
\end{tabular}

- = Significativo al nivel del $95 \%$

donde:
$\mathrm{r}=$ Coeficiente de correlación
$\mathrm{ECM}(\%)=$ Error cuadrático medio en porcentaje.
$\operatorname{DIFA}(\%)=$ Diferencia agregada en porcentaje.
DW = Estimador de Durbin-Watson.
$\mathrm{F}_{\mathrm{cal}} \quad=$ Valor $\mathrm{F}$ de la prueba de significancia
E.S. = Error estándar.
a. $\mathrm{b}=$ Coeficientes de la función. 
El análisis de varianza señala, además, que las funciones de volumen obtenidas explican muy bien los datos, ya que en todas las muestras el valor del $\mathbf{F}_{\mathrm{cal}}$ es mayor que el $\mathbf{F}_{\text {tabla. }}$ por lo que las variables independientes tienen influencia en la variable dependiente, haciendo estadísticamente significativos los modelos obtenidos.

Los valores obtenidos para el error cuadrático medio y la diferencia agregada, señalan que las propiedades estimadoras y la magnitud de los sesgos en los modelos obtenidos serían superiores en el área de Los Riscos y levemente inferiores en el área de Tanumé.

En las figuras $\mathrm{N}^{\circ} 5,6$ y 7 se presentan gráficamente los volúmenes reales versus los estimados, para el área de Los Riscos, Tanumé y Peñuelas, respectivamente.

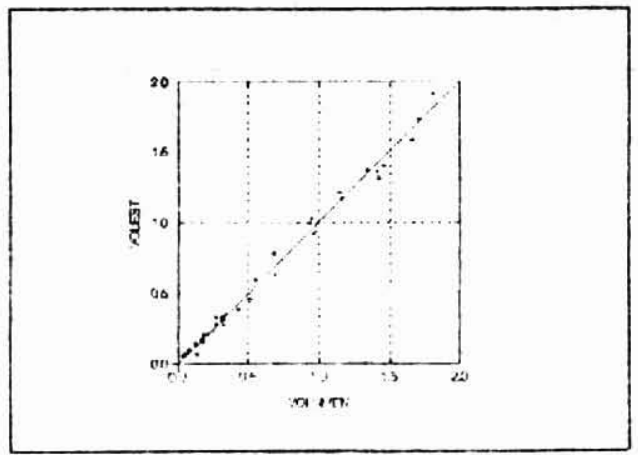

Figura N $N^{\circ}$ 5. RELACIÓN VOLUMEN REAL Y VOLLIMEN ESTIMADO ÁREA LOS RISCOS.

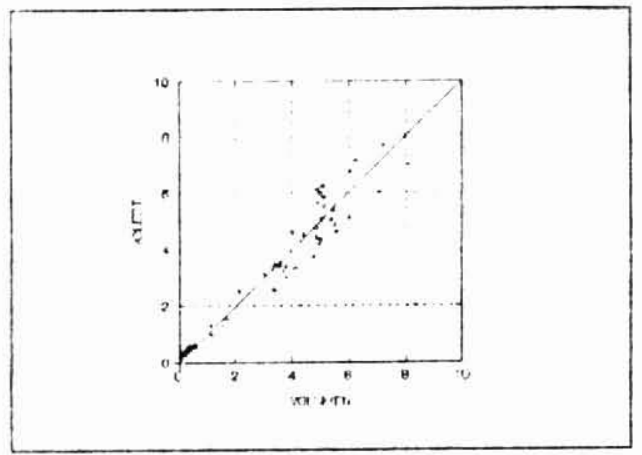

Figura $\mathrm{N}^{\circ}$ 6. RELACIÓN VOLUMEN REAL Y VOLUMEN ESTIMADO AREA TANUMÉ. 


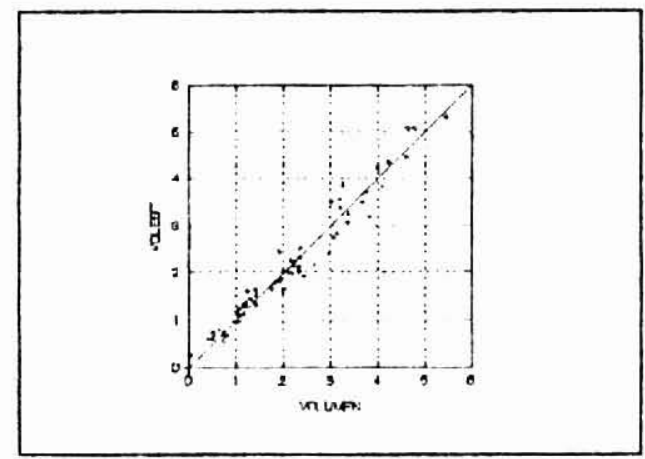

Figura $N^{\circ}$ 7. RELACIÓN VOLUMEN REAL Y VOLUMEN ESTIMADO ÁREA PEŃUELAS.

La bondad del ajuste de los modelos puede ser también apreciada a través de la regresión hecha entre los valores reales del volumen y los valores estimados. Los coeficientes de regresión obtenidos deben cumplir con ciertas hipótesis para asegurar que el ajuste es exacto y preciso. sin presentar sesgos.

La regresión se realiza ajustando un modelo lineal del tipo:

$$
V e=a+b * V o
$$

donde:

$\mathrm{Ve}=$ Volumen estimado

$V_{0}=$ Volumen observado.

$a \cdot b=$ Coeficientes de la regresión.

Si en las regresiones obtenidas el valor de la constante (a) es igual a cero, y el valor del término que acompaña a la variable independiente (b) es igual a 1, los ajustes son exactos y precisos. Esto significaría que existe una perfecta correspondencia entre el volumen estimado y el volumen real.

Para obtener la significancia estadística de los coeficientes de estas regresiones. los valores resultantes de las dócimas de hipótesis $\left(t_{\mathbf{a}}=\mathbf{0} ; \mathbf{t}_{\mathbf{b}}=\mathbf{1}\right)$ se deben comparar con los tabulares de la distribución $t$ de Student, fijando una región crítica para una probabilidad $\alpha=0,05$, con contraste bilateral. Si el valor calculado es menor que el valor crítico de $t$ para los correspondientes grados de libertad, (n-2), se infiere que la estimación proporciona la exactitud requerida. 
En el Cuadro $\mathrm{N}^{\circ} 4$ se presentan los valores de los coeficientes de regresión y su significación estadística para las tres áreas.

\section{Cuadro $\mathrm{N}^{\circ} 4$.}

BONDAD DE AJUSTE DE LOS MODELOS.

\begin{tabular}{|c|c|c|c|c|c|c|c|}
\hline \multirow[b]{2}{*}{ AREA } & \multicolumn{4}{|c|}{ COEFICIENTES DE REGRESIÓN } & \multirow[b]{2}{*}{$\mathrm{n}$} & \multirow[b]{2}{*}{$\begin{array}{l}\text { Valor } \\
t_{\text {tabla }}\end{array}$} & \multirow[b]{2}{*}{$r$} \\
\hline & a & $\begin{array}{c}\text { Valor } \\
\mathrm{t}_{\mathrm{cal}}\end{array}$ & b & $\begin{array}{c}\text { Valor } \\
\mathrm{t}_{\mathrm{cal}}\end{array}$ & & & \\
\hline TANUME & 0,13609 & 1,3456 & 0,952599 & 1,8125 & 65 & 1,9983 & 0,98 \\
\hline PENUELAS & 0,085723 & 1,3923 & 0,959256 & 1,6350 & 65 & 1,9983 & 0,98 \\
\hline LOS RISCOS & 0,004146 & 0,3919 & 0,993472 & 0.4840 & 42 & 2,0211 & 0,99 \\
\hline
\end{tabular}

donde:

$\mathrm{t}_{\text {cal }}=$ Valor de $\mathrm{t}$ calculado.

$\mathrm{t}_{\text {abla }}=$ Valor $\mathrm{t}$ de tabla para un $97,5 \%$ de probabilidad $\mathrm{y}(\mathrm{n}-2)$ grados de libertad.

$\mathrm{n} \quad=$ Número de observaciones del volumen en el área

$\mathrm{r} \quad=$ Coeficiente de correlación.

Los resultados del Cuadro $\mathrm{N}^{\circ} 4$ señalan que sólo en el área de Tanumé podría existir una leve sobreestimación para el caso de los volúmenes inferiores, la que sin embargo no es estadisticamente significativa. En el resto de las situaciones el análisis estadístico permite asegurar que el valor de "b" es similar a 1 , y el valor de "a" similar a 0 . La ausencia de sesgos importantes permite apreciar las buenas propiedades estimadoras de los modelos ajustados.

\section{Comparación Funciones de Regresión}

Al observar los modelos ajustados es posible comprobar la similitud de sus valores y comportamiento. Por ello, se intentó determinar si era posible utilizar la ecuación de Spurr, también llamada de variables combinadas, para explicar la totalidad de los volúmenes, considerando que la información procede de diferentes rodales y regiones.

En este estudio se utilizó para la comparación de los modelos de regresión el análisis de la covarianza, comparando las pendientes y términos independientes entre las funciones. El objetivo de este procedimiento es determinar si es posible combinar la información para obtener un modelo de regresión común para el conjunto de los datos.

Luego de los cálculos básicos para los análisis de varianza se realizó la comparación de las regresiones entre cada una de las áreas de estudio. obteniendo los siguientes resultados: 
Análisis área Tanumé y Los Riscos:

\begin{tabular}{|l|c|c|c|c|}
\hline \multicolumn{1}{|c|}{ Causa Variación } & $\begin{array}{c}\text { Suma de } \\
\text { Cuadrados }\end{array}$ & $\begin{array}{c}\text { Grados de } \\
\text { Libertad }\end{array}$ & $\begin{array}{c}\text { Cuadrado } \\
\text { Medio }\end{array}$ & F \\
\hline Regresión Combinada & 531,99433 & 1 & 531,99433 & $3.579 .205,49^{* *}$ \\
\hline Entre Pendientes & 0,7240754 & 1 & 0,7240754 & $4.871,508^{* * *}$ \\
\hline Entre Términos Ind. & 17,646838 & 1 & 17,646838 & $118.726,2^{* *}$ \\
\hline Residual & 0,0153094 & 103 & 0,000148 & \multicolumn{1}{|c}{} \\
\hline Total & 550,38056 & 106 & \multicolumn{2}{|l}{} \\
\cline { 1 - 4 } & &
\end{tabular}

** sefiala altamente significativo al nivel del $0.1 \%$.

\section{Análisis Área Peñuelas y Los Riscos:}

\begin{tabular}{|l|c|c|c|c|}
\hline \multicolumn{1}{|c|}{ Causa Variación } & $\begin{array}{c}\text { Suma de } \\
\text { Cuadrados }\end{array}$ & $\begin{array}{c}\text { Grados de } \\
\text { Libertad }\end{array}$ & $\begin{array}{c}\text { Cuadrado } \\
\text { Medio }\end{array}$ & F \\
\hline Regresión Combinada & 176.20452 & 1 & 176,20452 & $1890426,83^{* *}$ \\
\hline Entre Pendientes & 1,1214513 & 1 & 1,1214513 & $12031,597^{* *}$ \\
\hline Entre Términos Ind. & 4,5586528 & 1 & 4,5586528 & $48907,9376^{* *}$ \\
\hline Residual & 0,0096005 & 103 & 0,000009 & \multicolumn{1}{|c}{} \\
\hline Total & 181.89422 & 106 & \multicolumn{2}{|l}{} \\
\cline { 1 - 4 } & &
\end{tabular}

** señala altamente significativo al nivel del $0.1 \%$.

Análisis área Peñuelas y Tanumé:

\begin{tabular}{|c|c|c|c|c|}
\hline Causa Variación & $\begin{array}{c}\text { Suma de } \\
\text { Cuadrados }\end{array}$ & $\begin{array}{c}\text { Grados de } \\
\text { Libertad }\end{array}$ & $\begin{array}{c}\text { Cuadrado } \\
\text { Medio }\end{array}$ & $\mathrm{F}$ \\
\hline Regresión Combinada & 499.68422 & 1 & 499,68422 & $99.621,949^{* *}$ \\
\hline Entre Pendientes & 0.710439 & 1 & 0.710439 & $141,640084^{* *}$ \\
\hline Entre Términos Ind. & 22.025792 & 1 & 22,025792 & $4.391 .278^{* *}$ \\
\hline Residual & 0,6319914 & 126 & 0,0050158 & \\
\hline Total & 523.05244 & 129 & & \\
\hline
\end{tabular}

* seriala altamente significativo al nivel del $0.1^{\circ}$.

En todos los análisis los resultados demuestran que la regresión es altamente significativa. Igualmente significativa es la diferencia de pendientes entre las regresiones separadas. lo que implica que esta no es debida a los efectos aleatorios en el muestreo de los datos. En el caso de los términos independientes, en todos los análisis estos difieren significativamente. lo que indica que estadisticamente las regresiones son distintas.

Dado que existen diferencias entre los términos independientes, y que las regresiones presentan distintas pendientes. se llega a la conclusión que los datos de las tres áreas no pueden ser agrupados bajo un sólo modelo de regresión, en este caso el de 
variables combinadas, que tenga por objetivo la estimación de los volúmenes, sin una consecuente pérdida de exactitud.

\section{Modelo General de Volumen o Datos Combinados}

Aún cuando el análisis anterior concluyó que la función de Spurr no podía ser aplicable a toda la información en conjunto. sin una significativa pérdida de precisión. se realizó un ajuste utilizando otros modelos de volumen para todos los datos, con el objetivo de encontrar alguna otra función que sí sirviese para la estimación del volumen independiente del área de aplicación. Para ello se agrupó la información como si proviniesen de un sólo rodal. a la cual se les realizó el respectivo ajuste.

En la búsqueda de la mejor función de volumen se probaron diferentes modelos informados por la literatura. y algunos probados en estudios desarrollados anteriormente por INFOR. siendo las siguientes 5 funciones las que presentaron un mejor resultado:

A) $\operatorname{Ln} V=a+b^{*} \operatorname{Ln} D A P-c^{*} \operatorname{In} H-d^{*}(D A P)+e^{*} H$

B) $V \quad-D A P^{2} *\left(a-b^{*} H\right) \quad$ (Ogava)

C) $V \quad a-b^{*} \operatorname{Ln} D A P+c^{*} \operatorname{Ln} H+d^{*} D A P^{2}+e^{*}(I H)+f^{*}\left(D A P^{2} H\right)$

D) $\ln V^{*}=a^{*} D A P^{2}+b^{*}(I D A P)-c^{*} D A P^{2} * H+d^{*} H-e^{*}(1 H)$

E) $V=a^{*}(D A P)^{b}$

(Mever)

donde:

$i=$ Volumen $\left(\mathrm{m}^{3} \mathrm{ssc}\right)$.

DAP = Diámetro a la altura del pecho en $\mathrm{cm}$.

$H \quad=$ Altura total del árbol en metros.

$a, b, c, d, e, f=$ Coeficientes de las funciones.

En la figura $\mathrm{N}^{\circ} 8$ se presenta la distribución al agrupar todos los datos. mientras que en el Cuadro $\mathrm{N}^{\mathrm{N}} 5$ se exponen los respectivos valores de los indicadores de bondad de ajuste de los modelos probados. 


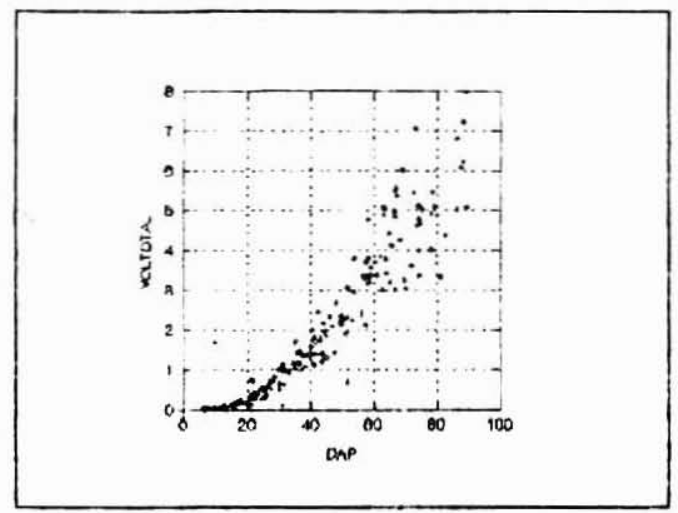

Figura $N^{\circ}$ 8. RELACIÓN DAPNOLUMEN TOTAL

Cuadro $\mathrm{N}^{\circ} 5$.

INDICADORES DE A.JUSTE DE LOS MODELOS

\begin{tabular}{|l|c|c|c|c|c|}
\hline \multirow{2}{*}{ INDICADOR } & \multicolumn{5}{|c|}{ MODELOS } \\
\cline { 2 - 6 } & $\mathrm{A}$ & $\mathrm{B}$ & $\mathrm{C}$ & $\mathrm{D}$ & $\mathrm{E}$ \\
\hline $\mathrm{r}$ & 0,97 & 0,99 & 0,98 & 0,97 & 0,96 \\
\hline $\mathrm{r}^{2}$ & 0,94 & 0,98 & 0,96 & 0,94 & 0,93 \\
\hline ECM (\%) & 17,8 & 21,6 & 27,4 & 21,3 & 37,2 \\
\hline DIFA (\%) & $-2,495$ & 6,019 & 0,697 & 1,231 & $-2,495$ \\
\hline $\mathrm{D}-\mathrm{W}$ & 2,206 & 1,539 & 1,647 & 2.286 & 1,919 \\
\hline $\mathrm{F}_{\text {cal }}$ & $747,17^{*}$ & $3.465,35^{*}$ & $809,88^{*}$ & $612,46^{*}$ & $2.232,27^{*}$ \\
\hline E.S. & 0,377 & 0,450 & 0,398 & 0,373 & 0,431 \\
\hline
\end{tabular}

- = Significativo al nivel del $95 \%$.

donde:

$\hat{r} \quad=$ Coeficiente de determinación.

$\mathrm{r}=$ Coeficiente de correlación

$\mathrm{ECM}(\%)=$ Error cuadrático medio en porcentaje.

$\operatorname{DIFA}(\%)=$ Diferencia agregada en porcentaje.

D-W = Estimador de Durbin-Watson.

$\mathrm{F}_{\mathrm{cal}} \quad=$ Valor $\mathrm{F}$ de la prueba de significancia 
E.S. = Error estándar.

El análisis de cada uno de los estimadores de la bondad de ajuste permite determinar cual de estos modelos presenta la mejor relación con los datos originales. De igual modo, el análisis gráfico del ajuste entre los valores observados y los valores estimados nos entrega otro elemento para la determinación de la precisión del modelo. En las figuras $\mathrm{N}^{\circ} 6,7,8,9$, y 10 se presentan los modelos con sus ajustes para los modelos A, B, C. D, y E, respectivamente.

En primer lugar, el coeficiente de correlación indica la relación entre los puntos de la muestra y el modelo obtenido. Asociado a este valor está el coeficiente de determinación, el que indica en que porcentaje la variación muestral de la variable dependiente puede ser atribuída a variaciones en la(s) variable(s) independiente(s).

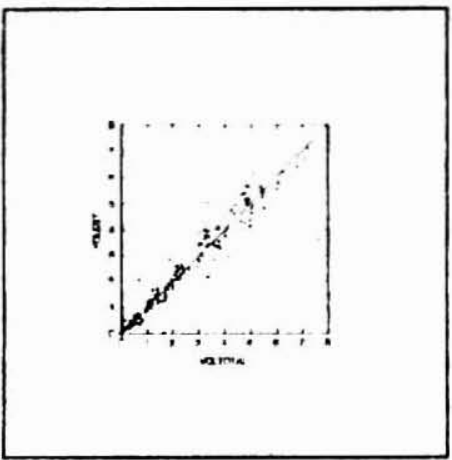

Figura $N^{\circ}$ 9. RELACIÓN VOLUMEN OBSERVADO Y VOLUMEN ESTIMADO MODELO A.

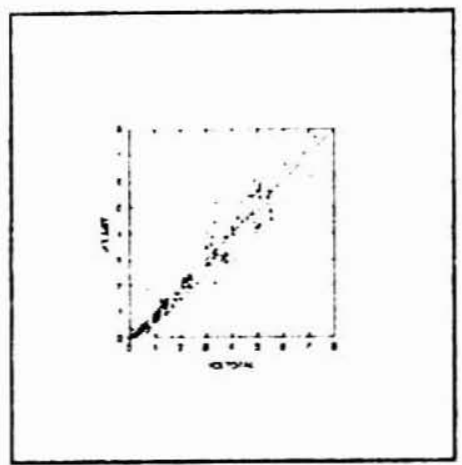

Figura $N^{\circ}$ 10. RELACIÓN VOLUMEN OBSERVADO Y VOLUMEN ESTIMADO MODELO B. 


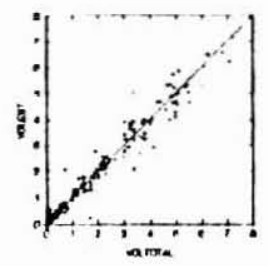

Figura $\mathrm{N}^{\circ}$ 11. RELACIÓN VOLUMEN OBSERVADO Y VOLUMEN ESTIMADO MODFLOC $\mathrm{C}$

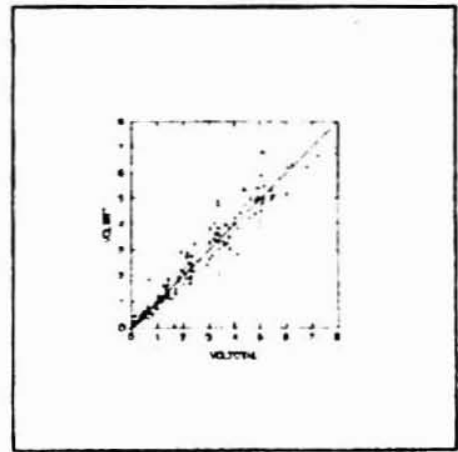

Figura $\mathrm{N}^{\circ} 12$. RELACIÓN VOLUMEN OBSERVADO Y VOLUMEN FSTIMADO MODELO $\mathrm{D}$.

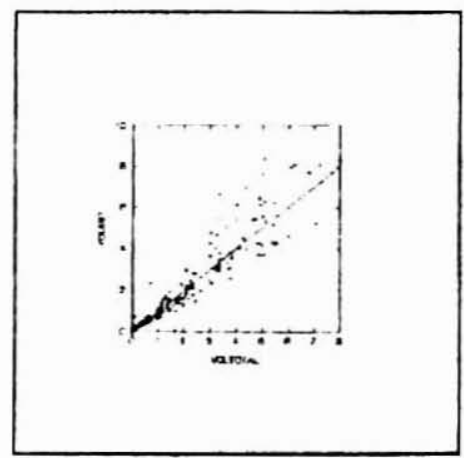

Figura $\mathrm{N}^{\circ}$ 13. RELACIÓN VOLUMEN OBSERVADO Y VOLUMEN ESTIMADO MODELO $\mathrm{E}$.

Tal como se aprecia en el Cuadro $\mathrm{N}^{\circ} 5$. en los 5 modelos seleccionados el valor del coeficiente de correlación fue alto. $(0.96$ a 0.99$)$. mientras que el coeficiente de 
determinación varió entre 0.93 y 0.98 . Esto señala que todos los modelos presentaron una alta relación entre los valores reales y los estimados. Sin embargo, este indicador por sí sólo no basta para concluir sobre la bondad del modelo, pudiendo existir un coeficiente alto pero un mal ajuste.

Este coeficiente de correlación está relacionado, además. con el error estándar de la estimación. el cual mide la dispersión de los puntos con respecto del modelo obtenido, y de acuerdo a los resultados del estudio, este no fue superior a 0.450 (modelo B).

El analizar la correlación serial (autocorrelación) de los residuos del modelo, valor de Durbin-Watson, tiene por objetivo asegurar que los errores a nivel muestral (residuos) no tengan ningún grado de dependencia entre sí para distintos $\mathrm{X}$. Cuando este estimador está entre 1.6 y 2 no existe autocorrelación. En este estudio los valores de este estimador están cercanos al margen donde no existe autocorrelación, con la excepción del modelo $\mathrm{B}$ (1.539).

El análisis de varianza realizado señala, además. que los modelos de volumen propuestos explican muy bien los datos. ya que en todos los casos el valor del $\mathbf{F}_{\text {cal }}$ es mayor que el $\mathbf{F}_{\text {tabla. }}$. por lo que las variables independientes tienen influencia en la variable dependiente. haciendo estadisticamente significativos los modelos obtenidos.

Sin embargo. si se asume que el ECM y la DIFA coinciden con las propiedades estimadoras del volumen total, y considerando además que en las estimaciones agregadas la magnitud de los sesgos es un factor importante. los modelos seleccionados no presentarian un ajuste adecuado ni similar entre ellos.

Aún cuando los valores del cocficiente de correlación son altos. y existe una aceptable correlación serial de los residuos. los valores del ECM y DIFA nos señala que en general los modelos ajustados no presentan adecuadas capacidades estimativas, ratificando que para las tres áreas no es aconsejable el uso de un sólo modelo de volumen.

Al observar el conjunto de valores de cada uno de los estimadores utilizados. se puede considerar que los modelo $\mathrm{B}$ y D presentan valores y comportamientos similares. mientras que el modelo $\mathrm{A}$ tiene los mejores valores de los diferentes indicadores de la bondad del ajuste.

$\mathrm{El}$ análisis de la relación entre los distintos valores del Cuadro $\mathrm{N}^{\mathrm{0}} 5$, permite concluir que. con la salvedad del modelo A. los modelos no serían eficientes en la estimación del volumen. El modelo A podría ser utilizado. pero dada su estructura logaritmica presenta una subestimación constante del volumen.

Esta falta de estimación puede deberse a las diferencias de crecimiento encontradas en las tres áreas de estudio. con la presencia de árboles que por sus características no permiten ser asociados con el resto de los individuos a través de un solo modelo 
predictor. Estas situaciones pueden ser mejor analizadas mediante el uso de modelos individuales, obteniendo una mayor precisión en cada área de estudio.

\section{DISCUSIÓN Y CONCLUSIONES}

La determinación del volumen de los árboles o parte de él, es una de las informaciones importantes en la descripción de las masas forestales, en donde este valor permite apreciar la ganancia volumétrica producida al combinar los efectos del diámetro y la altura.

El desarrollo de modelos matemáticos que utilizan como variable dependiente el volumen, y como independientes variables de estado del árbol. como lo son el DAP y la altura total y/o alguna otra variable sencilla de cuantificar. como así también diversas transformaciones de ellas. han permitido una mayor facilidad en la estimación de este parámetro.

En el marco de este estudio si bien existe una alta variabilidad en la información original ( 3 áreas geográficas y diferentes edades). las funciones obtenidas permiten contar con modelos aceptables para estimaciones volumétricas con un alto grado de confiabilidad.

Los valores obtenidos en los ajustes permiten concluir que la ecuación de Spurr (1952) parece representar razonablemente bien el comportamiento volumétrico en las tres áreas en estudio.

La aplicación de esta función sobre los datos del estudio. comparando los volúmenes reales medidos con los correspondientes estimados, permitió establecer las propiedades estimadoras aceptables de los modelos para $\mathbf{E}$. globulus.

La bondad del ajuste de los modelos puede ser también apreciada a través de la regresión hecha entre los valores reales del volumen y los valores estimados. En las regresiones obtenidas los resultados señalan que sólo en el área de Tanumé podría existir una leve sobreestimación para el caso de los volúmenes inferiores. En el resto de las situaciones el valor de "b" es similar a 1 . y el valor de "a" similar a 0 . La ausencia de sesgos importantes confirma las buenas propiedades estimadoras de los modelos ajustados.

Al intentar explicar los volúmenes mediante una función común por medio del análisis de la covarianza. comparando las pendientes y términos independientes de las tres funciones obtenidas. se llega a la conclusión que las tres áreas no pueden ser agrupados en un sólo modelo de regresión que tenga por objetivo la estimación de los volúmenes. sin una consecuente pérdida de exactitud. 
De igual forma, el ajustar otros modelos para la estimación del volumen independiente del área de aplicación agrupando los datos como si proviniesen de un sólo rodal, permite concluir que los modelos estudiados en general no serían eficientes.

Uno de los modelos seleccionados presenta valores aceptables para los indicadores de la bondad del ajuste (modelo A), sin embargo, en el uso de esta función se debe considerar que por su estructura logarítmica presenta una subestimación constante del volumen.

Esta falta de estimación puede deberse a las diferencias de crecimiento encontradas en las tres áreas de estudio, con la presencia de árboles que por sus características no permiten ser asociados con el resto de los individuos a través de un solo modelo predictor. Estas situaciones pueden ser mejor analizadas mediante el uso de modelos individuales, obteniendo una mayor precisión en cada área de estudio.

Los modelos obtenidos por área geográfica son confiables en sus estimaciones. Sin embargo. se debe considerar que estos tienen como base rodales adultos de $\mathbf{E}$. globulus, por lo que serían de utilidad en la estimación de volúmenes aserrables o para la produccion de chapas.

El poder contar con un modelo para volumen que sea utilizable en rodales adultos para cualquier zona de crecimiento del E. globulus en el pais, dependerá de una mayor cantidad de nueva información, de las propias necesidades de herramientas para la predicción forestal, $y$ de las edades de rotación utilizadas.

\section{REFERENCIAS}

Bennet, F.A., Swindel, B.F., 1972. Taper Curves for Slash Pine. USDA Forest Service Research. Note SE-179.

Bruce, D., Curms, B. and Vancaevering, H.. 1968. Development of a Sistem of Taper and Volume Tables for Red Alder. For. Sci. 14(3) : 339-350.

Cao, Q. V., Burkhart H.E., and Max T.A., 1980. Evaluation ot Two Methods for Cubic - volume Prediction of Loblolly Pine to Any Merchantable Limit. For. Sci. 26(1) : 71-80.

Draper, N. and Smith, H.. 1967. Applied Regression Analysis. John Wiley and Sons Inc., New York. 407 p.

FAO. 1980. Estimación del Volumen Forestal y Predicción del Rendimiento. Vol. 22/1 y $22 / 2$. Roma. 80 p.

Freese, P.. 1960. Testing Accuracy. Forest Service. Washington D.C. Volume 6 (2). 
Husch, B., Miller, C., and Beers, T., 1982. Forest Mensuration. Third edition. The Ronald Press Company. New York. 402 p.

INFOR. 1991. Eucalyptus, Principios de Silvicultura y Manejo. CORFO-INFOR. Santiago. 197 p.

Kerruish, C. and Rawlins. W., 1991. The young eucalypt report. CSIRO, Australia, $272 \mathrm{p}$.

Ostle, B., 1977. Estadística aplicada. México. Ed. Limusa-Willey. 628 p. 